Instituto Internacional de Investigación y Desarrollo Tecnológico Educativo INDTEC, C.A.

DOI: https://doi.org/10.29394/scientific.issn.2542-2987.2017.2.5.20.377-395

OAI-PMH: http://www.indteca.com/ojs/index.php/Revista Scientific/oai

\title{
Hermeneusis Ontológica del Estudiante Universitario
}

\author{
Autora: Mariela Eduvigis Jiménez Campos \\ Universidad Centroccidental "Lisandro Alvarado", UCLA \\ mjimenez@ucla.edu.ve \\ Lara, Venezuela
}

\section{Resumen}

La educación universitaria debe promover una enseñanza dirigida a mediar en cada estudiante el desarrollo pleno de sus potencialidades humanas durante su formación académica, para que los discentes puedan conocerse, valorarse, reconocerse como personas, ciudadanos y futuros profesionales y así logren alcanzar su autorrealización plena. El propósito de este ensayo es hacer una hermeneusis ontológica del estudiante universitario. La metodología utilizada se basó en la revisión documental de los aspectos teóricos constitutivos de los constructos filosóficos expuestos por Husserl, Heidegger y Gadamer, entre otros autores, con la finalidad de conocer e interpretar el Dasein estudiantil, desde los elementos que conforman su capacidad biopsico-social-cultural que les permite desarrollarse como profesionales desde una concepción de ciudadanos integrales capaces de asumir la realidad como actores que están ahí en tiempo y lugar para transformarse en protagonistas de su propia historia. Finalmente, la hermeneusis de la ontología del estudiante universitario, puede entenderse como una formación académica integral que conlleva a desarrollar la autonomía para desempeñar el ejercicio profesional, con espontaneidad, independencia, idoneidad y firmeza de carácter, hacia el cumplimiento de sus metas personales que contribuyan a las soluciones que demanda la sociedad contemporánea.

Palabras clave: hermeneusis ontológica; estudiante universitario; dasein. 


\title{
Ontological Hermeneusis of the University Student
}

\begin{abstract}
University education must promote teaching aimed to mediate the full development of each student human potentialities during the academic training, so the students can know, value, recognize themselves as persons, citizens and future professionals and thus, they can achieve their complete selfrealization. The purpose of this essay is to perform an ontological hermeneusis of the university student. The methodology was based on the review of theoretical aspects constituting the philosophical constructs exposed by Husserl, Heidegger and Gadamer, among others, with the purpose of knowing and interpreting the student Dasein from the elements that make up their biopsycho-social-cultural capacity which allows them to develop as professionals from a conception of integral citizens capable of assuming reality as actors who are there in time and place to become their own history main characters. Finally, the hermeneusis of the university student ontology can be understood as a comprehensive academic training that leads to develop the autonomy to carry out the professional performance with spontaneity, independence, suitability and determination, toward the fulfillment of their personal goals that contribute to the contemporary society solutions.
\end{abstract}

Keywords: ontological hermeneusis; university student; dasein. 


\section{Preámbulo del Discurso}

La universidad en la búsqueda, desarrollo y divulgación del conocimiento en todos los campos del saber debe cumplir con las funciones de docencia, extensión, investigación y gestión, desde las cuales pueda asumir el proceso académico que coadyuve al mejoramiento del potencial humano para atender las necesidades del entorno laboral a partir de la formación profesional en el contexto del siglo XXI.

La educación universitaria debe responder a los requerimientos de una sociedad impregnada por las exigencias de los avances científicos, tecnológicos, económicos, entre otros, que impactan la realidad de la formación profesional, desde una perspectiva fenomenológica capaz de interpretar la complejidad de la dinámica humana que se desarrolla en los contextos socio-laborales cada día más globalizados.

La globalización en la educación universitaria representa un elemento dinamizador de la sociedad postmoderna, en el aprovechamiento de las oportunidades comunicativas que provee la información en congruencia con el desarrollo del talento humano que requiere de la conformación de habilidades, destrezas, actitudes, sobre una base curricular más eficiente, efectiva y eficaz, en respuesta a las exigencias de la calidad en el contexto de la sociedad del conocimiento.

Vista de esta manera, la calidad educativa, debe enfocarse en la eficacia, coherencia y pertinencia social, las cuales representan premisas dirigidas a brindar a los estudiantes universitarios una formación que les proporcione las herramientas necesarias para consolidar el perfil profesional de los ciudadanos, que les permita el fortalecimiento de conocimientos tecnocientífico-humanístico en el momento histórico requeridos por la aldea global.

El propósito de este ensayo es reconfigurar desde una hermeneusis ontológica del estudiante universitario, los elementos constitutivos de su potencial bio-psico-social-cultural que los conlleva a formarse como 
profesionales desde una concepción de ciudadanos integrales, capaces de asumir la realidad como actores que están ahí en tiempo y lugar para ser protagonistas de su propia historia en la sociedad contemporánea.

\section{Constructo Teórico del Discurso}

La universidad brinda al estudiante la oportunidad de explorar una realidad vivencial que representa, además de su formación académicaprofesional, una oportunidad de independizarse de su familia; lo que implica, en algunas ocasiones, alejarse de su hogar para comenzar una nueva etapa. En este sentido, las funciones de docencia, extensión, investigación y gestión forman parte esencial del proceso formativo que coadyuva a potenciar el talento humano, para atender las necesidades del entorno académico-laboral desde la formación profesional, en respuesta a los requerimientos de la sociedad.

La Organización de las Naciones Unidas para la Educación la Ciencia y la Cultura (UNESCO, 2005), en su Informe de Seguimiento de Educación para Todos en el Mundo, señala que la educación universitaria, se caracteriza por dos principios: a). el desarrollo cognitivo del estudiante, lo que significa el objetivo más importante de todo sistema educativo y su éxito es un indicador de la calidad de la educación que ha recibido; b). el fomento de actitudes y valores concordantes con una buena conducta cívica y la creación de condiciones favorables para el desarrollo afectivo y creativo del estudiante.

Las competencias del estudiante universitario, de acuerdo a la Agencia para la Calidad del Sistema Universitario de Cataluña (2003), son aquellas que deben contemplar habilidades (a) académicas, que demuestran en su formación teórica y práctica); (b) instrumentales, que se expresan en la capacidad de gestión de idiomas, informática, entre otras; (c) interpersonales, explicitadas en la expresión oral y escrita, el liderazgo, el trabajo en equipo, 
entre otros aspectos; y (d) cognitivas, que les permite la toma de decisiones, pensamiento crítico, razonamiento cotidiano, creatividad.

Por consiguiente, la educación universitaria debe estar enfocada en el desarrollo que sea sostenible en el tiempo y, de esta manera, contribuya al crecimiento integral de los estudiantes. Además, debe procurar en su currículo formativo la promoción de competencias personales y profesionales, lo que exige a los docentes el despliegue de su quehacer académico para alcanzar la comprensión del fenómeno estudiantil.

En esta intención comprensiva, constituye un punto de partida ineludible la interpretación que el estudiante hace de su propia realidad, a los efectos de lo cual, la fenomenología ofrece una vía de abordaje que facilita el acercamiento a la autopercepción de la esencia estudiantil. La fenomenología intenta develar las estructuras esenciales de la conciencia, focalizar la descripción, análisis e interpretación de los contenidos dados a conocer por los estudiantes universitarios en la condición subjetiva del mundo social, que de acuerdo a Husserl (1997), toma su significado y amplía sus significantes, en la manera como la piensan y viven.

Por otra parte, cuando se trata de la hermeneusis del fenómeno estudiantil, Heidegger (2005), se refiere al Dasein o "ser-ahí", como una condición primaria de carácter histórico que se desarrolla ontológicamente desde múltiples realidades diversas, debido a que se mueve dentro de una dinámica compleja e incierta de su existencia personal, de un educando que se forma como futuro profesional dentro de un contexto socio-histórico-cultural que le permite sentir y percibir el escenario universitario desde sus propias vivencias.

Desde la filosofía existencialista, Heidegger (op.cit.) sostiene que el Dasein, se da como una forma de interpretación y comprensión de las concepciones internas del estudiante como ser humano, de cómo se percibe y toma conciencia plena de su realidad desde la autorreflexión, la cual se 
encuentra libre de convencionalismos. De igual modo, replantea la pregunta sobre la propia existencia humana, que busca la razón del ser, y sostiene que la filosofía no puede lanzarse a ciegas sobre él, olvidando las condiciones bajo las cuales su sentido es buscado desde el razonamiento de que podría ser encontrado.

Por otra parte, Ugas Fermín (2013) define la realidad como "una interpretación de lo real-existente. De manera que lo Real es lo que está siendo, y no lo que pensamos debe ser" (pág. 30). Es decir, que el entorno del estudiante es percibido de acuerdo a las experiencias y relaciones comunicativas que conforman el Dasein existencial de las vivencias de ellos en el "aquí y en el ahora" de su vida universitaria.

La hermeneusis, como fundamento epistemológico, argumenta que la comprensión del ser es una condición humana natural. Constituye la experiencia de vida que busca interpretar para comprender su propia realidad en la cotidianidad de las vivencias estudiantiles. En esta orientación, Schwandt, (1997) se apoya en la hermenéutica filosófica de Gadamer (2004). El Dasein es una visión que se ubica en estar - ahí - en - el - mundo, lo que implica que el estudiante universitario, no está separado de la experiencia lingüística del entorno histórico-socio-cultural; por lo que se presupone es un ser - en - el - lenguaje, lo cual le permite interactuar con otros. Asimismo, refiere que el comprender es interpretar, y todo acto interpretativo sucede como enfoque que permite la comprensión interpretativa de los significados.

La hermeneusis se desarrolla en un contexto espacial y único que se vincula a las experiencias comunicativas del estudiante universitario, que constituye un foco inteligible del conocimiento y de la existencia humana, lo que reconoce en el lenguaje las experiencias que cada quien tiene en el mundo existencial que, de acuerdo a Gadamer (op.cit.), debe entender "el todo desde lo individual y lo individual desde el todo (...) El movimiento de la comprensión discurre así del todo a la parte y de nuevo al todo" (pág. 63). 
En tal sentido, la concepción filosófica de hermeneusis de Heidegger (op.cit.), sostiene que "la conciencia se manifiesta, por consiguiente, como una atestiguación perteneciente al ser del Dasein, en la que el Dasein es llamado ante su propio poder-ser" (pág. 307). La trascendencia del estudiante, deriva de la comprensión del ser en la realidad universitaria, que se explica en la expresión de los testimonios que sirven para aclarar la interpretación existencial en el contexto académico de su formación profesional, que no es una invención, sino una construcción ontológica, cuyo fundamento es el bosquejo de sus rasgos elementales.

La ontología estudiantil de acuerdo con Echeverría (2013), propone la necesidad de vincular el aprendizaje con una acción formativa transformadora, puesto que, sin darse cuenta, los docentes como académicos, han ido desvinculando la praxis pedagógica con los demás propósitos de la vida sociolaboral. No obstante, el estudiante al ingresar a la universidad para formarse como profesional es concebido como un ente bio-psico-social-cultural, con un Dasein enmarcado en su propia realidad histórica como se puede observar en la imagen 1.

Imagen 1. Estudiante Universitario.

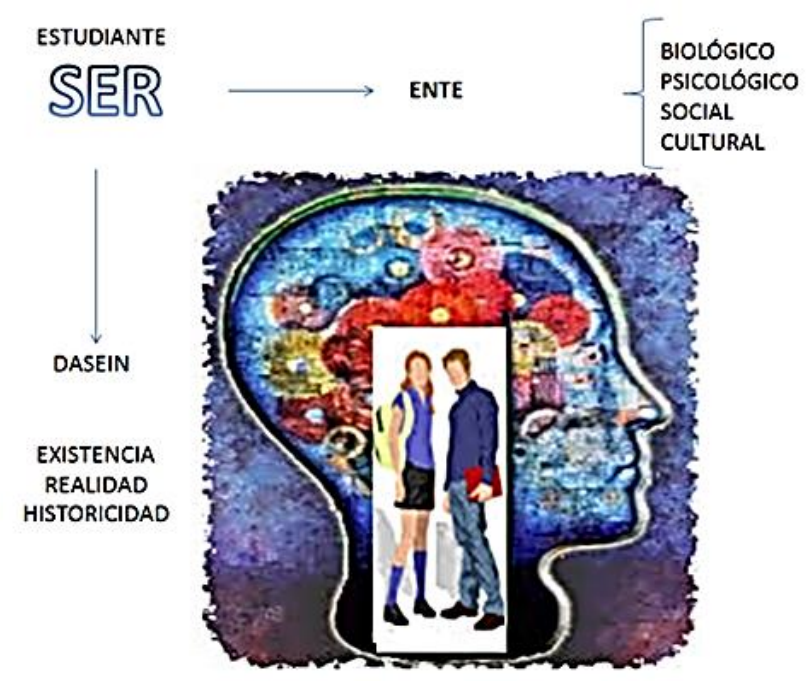

Fuente: Jiménez, 2017. 
En la realidad del estudiante universitario representada en la imagen anterior, el ser se divide en dos elementos fundamentales: el Dasein (el "serahí") como ente existencial "arrojado" a un contexto tangible, en el cual vive sus propias experiencias y el ser como ente bio-psico-socio-cultural, el cual se desenvuelve en dicha realidad.

En palabras de Heidegger (op.cit.), el Dasein se desenvuelve existencialmente en una realidad cambiante y complicada donde se desarrolla ontológicamente a partir de sus múltiples vivencias durante su formación como futuro profesional. De esta manera, cobra fuerza la significación del fenómeno de la ontología del estudiante universitario, debido a la conectividad de los significados que permitirán entrever las nuevas estructuras fundamentales del ser-ahí que es el fenómeno de estudio en esta investigación.

Así, cuando se habla de la realidad del estudiante universitario desde una posición filosófica, éste se convierte en un fenómeno de estudio, debido a las características mencionadas por Heidegger (op.cit.), que lo hacen un sistema de relaciones, en las cuales se asume como un ente intramundano que se proyecta en sus posibilidades de hacer una relectura de sus vivencias en el ámbito que es objeto del quehacer académico, porque él está ahí en una situación evidentemente existencial.

Desde el punto de vista Heideggeriano, el mundo de los seres en el cual fuimos arrojados, solo puede ser comprendido a la luz de la existencia y la inexistencia, el Ser y la Nada. El Ser dentro de sus posibilidades de existencia debe reconocer la diversidad cultural inherente a lo humano. Cuando los valores presentes en todas las culturas como: el respeto, la dignidad y la responsabilidad del ser humano son aplicados, se concilia con el auténtico humanismo.

Con referencia a lo anterior, Morín (2000) expresa que la educación universitaria del futuro deberá ser una educación universal basada en la condición humana. Es decir, la sociedad avanza a un ritmo vertiginoso en esta 
era planetaria, lo cual exige el surgimiento de un nuevo humanismo, donde los métodos y las estrategias de acción puedan ser aplicados al redescubrimiento de los ámbitos espirituales. De esta manera Ji (2011) explica que:

La inteligencia, en vez de hacer correr al hombre los riesgos que engendran sus suposiciones y usurpaciones, utilizará con creces su fuerza y vivacidad para servir la causa del hombre ahora sí realmente completo, pues no será ya un hombre desviado hacia lo material por lo cerebral, sino un hombre unificado, en cuerpo y alma, para hacer frente a su misterio por medio de su conciencia (pág. 27).

Es evidente, entonces, que el nuevo humanismo demanda del hombre, además de adquirir conocimientos, desarrollar la parte espiritual; en otras palabras, combinar la lógica con el afecto y la compasión. El gran filósofo Confucio consideraba que ser benevolente era el acto de amar a la gente y la compasión era el comienzo de la benevolencia.

Los retos que se presentan a los estudiantes universitarios en la realidad de la vida académica, como seres y entes estudiantiles, tienen implicaciones en su formación, que les exigen utilizar sus capacidades personales, intelectuales y emocionales, de acuerdo con los niveles de racionalidad, para que puedan adaptarse a un ambiente de aprendizaje que les posibilite desafiar las situaciones que enfrentan, en función de sus expectativas y de quienes los rodean.

De igual manera, la hermeneusis de la ontología del estudiante universitario, considera al ser como un ente bio-psico-socio-cultural, que se explica en la expresión de las vivencias estudiantiles surgidas de los testimonios de los docentes que intervienen en su formación profesional, los cuales permiten la comprensión interpretativa para una construcción ontológica cuyo fundamento es el bosquejo de sus manifestaciones elementales de su propia historia. 
En esta orientación, Engel (citado por Juárez, op.cit.), presenta un modelo biopsicosocial con una representación relacional de la jerarquía de sistemas, la cual se muestra en la imagen 2.

Imagen 2. Jerarquía de Sistemas De Engel GL.

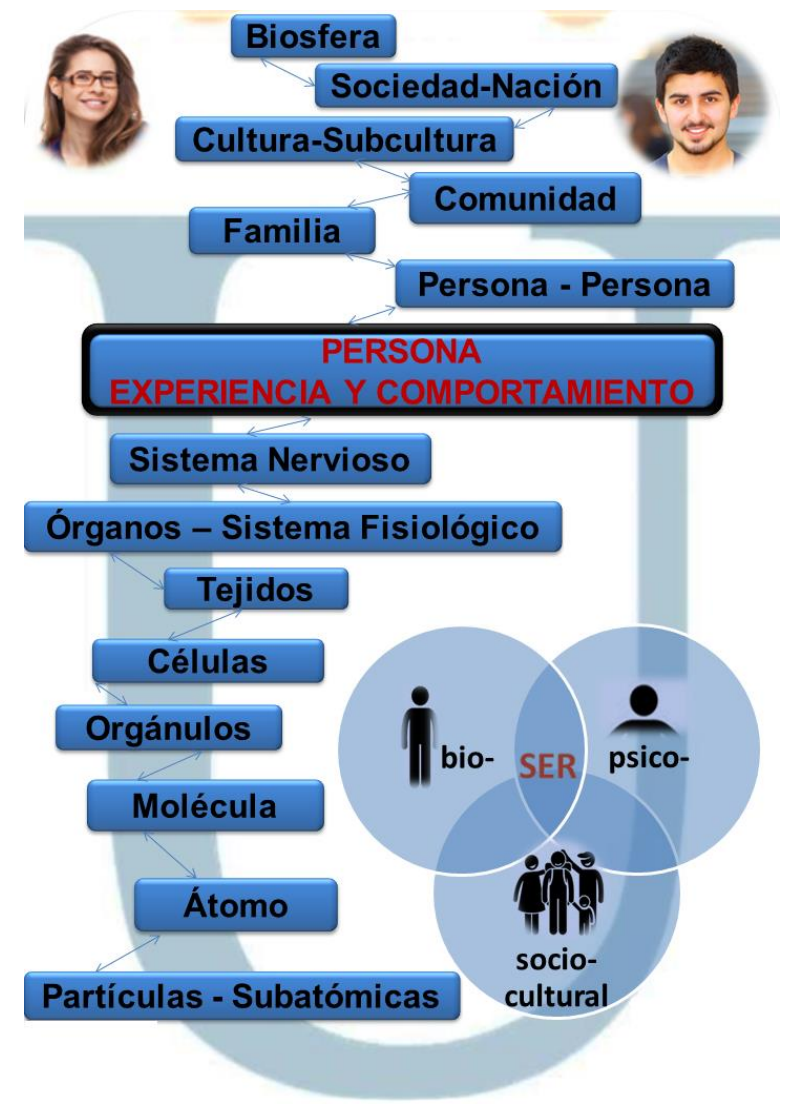

Fuente: Perales, Mendoza y Ortiz (2000). Adaptación: Jiménez, 2017.

En este modelo jerárquico, se puede observar la dimensión biológica en su integración con la dimensión psicológica, la cual está representada por las experiencias que cada individuo vivencia y procesa desde las experiencias sociales de sus interrelaciones significativas con su entorno familiar, que determinarán los rasgos básicos de su personalidad. En este modelo se pone de manifiesto que todo ser humano es un conjunto armonioso y funcional resultante de la interacción de los factores biológicos, psicológicos y sociales, 
que aportan al individuo características únicas para existir e interactuar en los diversos contextos.

Son precisamente las vivencias pasadas de cada realidad personal y grupal, las que representan la historia que, como ejercicio hermenéutico ideográfico, Heidegger (op.cit.), explica como un acontecer pasado que en el presente es el punto de referencia para la interpretación del mismo. Así. se propone la historiografía, que se inscribe en el dominio del pensamiento como representación de las vivencias, de acuerdo con la comprensión de cada estudiante de su propia realidad en el ámbito universitario a partir de sus experiencias previas.

Asimismo, el estudiante universitario es por naturaleza un ente biopsico-social, esto implica que por un lado, es un ser biológico que posee una parte objetiva y material: genes, células, órganos, sistemas, sangre, entre otros. Por otro lado, está presente el factor psicológico (parte intangible e inmaterial), determinado por los pensamientos, sentimientos, instintos, el comportamiento y las emociones. De este modo, el estudiante, como ser humano, tiene conciencia, estructuración de pensamiento y valor ético de sus actos personales íntimos y como ser biológico y social, desarrolla actos gregarios, a partir de los cuales busca a otros de la misma especie dentro de su entorno para convivir.

Visto de esta manera, el estudiante universitario como ser bio-psicosocial es un sistema abierto, dinamizado de adentro hacia afuera, es decir, desde lo individual, interactúa en un entorno de relaciones sociales de forma recíproca, de un ser que vive sus propias experiencias a partir de la interacciones internas y externas complejas.

En este contexto de complejidad dinámica e incierta de la realidad estudiantil, Morín (2000) señala que "la hominización desemboca en un nuevo comienzo. El homínido se humaniza. Desde allí, el concepto de hombre tiene un doble principio: un principio biofísico y uno psico-socio-cultural, ambos 
principios se remiten el uno al otro" (pág. 25). Es así como, desde la perspectiva de este autor, existe el factor influenciado por elementos externos: ambiente, sociedad, historia; sometido a un constante aprendizaje dinámico del entorno que lo rodea, donde gradualmente aprende y hace suya la cultura organizacional universitaria.

Al asumir la cultura organizacional universitaria, el estudiante comparte su historia y desarrolla el sentido de pertenencia con el ámbito académico, desde la socialización de sus relaciones con sus pares, pero también con los docentes y demás miembros de la comunidad estudiantil.

La comunidad estudiantil es un ámbito de interacciones socioculturales, en la cual, el estudiante universitario se desarrolla como un ser integral que de acuerdo a López (2013) requiere de:

Otra identidad y otra educación, para ser hoy ciudadano del mundo. Aquella educación, aquella fuente activa del ser, capaz de generar otra identidad psico-cultural, otro tipo de persona, que siendo un ser bio-psico-sociocultural complejo pueda afrontar con solvencia los retos de nuestro tiempo (pág. 73).

Según el criterio de López (op.cit.), la educación universitaria es un elemento complementario para el desarrollo de las potencialidades del estudiante como un individuo que puede seguir creciendo en lo personal, profesional y desarrollarse como un ciudadano apto para enfrentar los desafíos presentes y futuros en los diferentes ámbitos de su vida.

Con referencia a lo anterior, Silas (2011), expone que el crecimiento profesional es aquel que no se agota con la posibilidad de adquirir las habilidades y competencias de su área profesional, sino que permite también desarrollar un ser humano capaz de trascender su propia existencia para dar sentido a su vida personal, social y cultural con compromiso y entusiasmo en la búsqueda de su autorrealización. 
En tal sentido, Malavé de Zabala (2001), señala que un estudiante universitario exitoso se caracteriza por: a). tener una alta motivación intrínseca que involucra una red de conexiones cognitivo-afectivas, que conllevan un uso energizante de capacidades, destrezas para beneficio personal y del colectivo; b). asumir plena responsabilidad por el resultado de sus acciones; c). poseer una actitud favorable, así como un conocimiento pleno de la carrera que escogió; y d). ser responsable de su aprendizaje, hábitos de estudio para aprender a percibir, recordar, actuar, sentir y pensar en forma determinada bajo condiciones especiales.

Se puede decir que la excelencia estudiantil universitaria depende de una serie de comportamientos y acciones donde la motivación, actitud, responsabilidad sobre la propia educación, aunado al deseo del estudiante de alcanzar los objetivos establecidos juegan un papel primordial. En este contexto, el docente por su cercanía con el estudiante, representa la alternativa más viable de apoyo durante todo el trayecto universitario. Es desde su perspectiva, experiencia, formación, el idóneo para enrumbar, orientar, fomentar y apoyar el aprendizaje humanístico.

La condición humanística de la educación universitaria, de acuerdo al criterio de Salas (2000), no agota la posibilidad de crecimiento profesional, únicamente en el desarrollo de las habilidades y competencias de su área, sino que busca desarrollar un ser personal en beneficio de la trascendencia, del sentido de la vida, del compromiso, entusiasmo, sentido de identidad, de la vivencia de los valores humanos que le permiten su autorrealización.

De allí que el estudiante, está en la búsqueda de su autorrealización, de adquirir sabiduría, aprendizajes, a través del aprender a aprender, que se conviertan en las competencias para asumir su vida con sentido de responsabilidad, solidaridad social, respeto por el ambiente natural que lo rodea, para una transformación positiva de su entorno. Desde la visión de Salas (op.cit.): 
La superación profesional constituye un conjunto de procesos educacionales que posibilitan a los graduados universitarios la adquisición y perfeccionamiento continuo de los conocimientos y habilidades requeridos para un mejor desempeño en sus responsabilidades y funciones laborales (pág. 15)

Esto significa que un profesional competente es el resultado de un estudiante, que ha sido formado integralmente, quien luego de un aprendizaje holístico, será capaz de trasferir todo lo aprendido en la universidad al campo laboral, personal, cultural y social, con la potencialidad de reconocerse así mismo, para empoderarse de sus habilidades, destrezas y competencias, que le permitirán transformar su realidad.

Sin embargo, la formación holística estudiantil no es una labor sencilla, puesto que implica no sólo asumir un planteamiento curricular, sino fomentar en la estructura institucional los componentes necesarios para el desarrollo integral del estudiante universitario. Dicha tarea puede lograrse más eficaz y eficientemente, si los diversos actores universitarios contribuyen con los elementos fundamentales reflejados en la filosofía de gestión universitaria (visión, misión, estrategias y valores) a través de políticas educativas que fomenten el desarrollo holístico estudiantil.

En consecuencia, es indispensable realizar una verdadera hermeneusis de la ontología del estudiante universitario; asimismo, las universidades junto a sus actores sociales deben plasmar en sus aulas el interés por la formación de seres integrales donde, además, se ejecute la labor docente con amor. Lo anterior, podría coadyuvar para evitar o aminorar el índice de estudiantes sancionados y/o retirados de la universidad.

\section{Reflexiones Conclusivas del Discurso}

Los planteamientos desarrollados en este ensayo, permiten afirmar que la educación universitaria debe promover una enseñanza dirigida a mediar en 
cada estudiante el desarrollo pleno de sus potencialidades humanas en su formación académica, para que los discentes puedan conocerse, valorarse, reconocerse como personas, ciudadanos y futuros profesionales para que de esta manera logren alcanzar su autorrealización plena.

En tal sentido, las universidades durante la formación profesional generan conocimientos científicos, tecnológicos, culturales que deben influir de manera positiva en la dinámica personal, laboral de los contextos de convivencia cotidiana, que contribuyan con el orden social y el crecimiento del país. De igual forma, en este cometido educativo, el quehacer académico debe asumir con mayor profundidad, responsabilidad, preparación y participación los saberes científico-técnico-humanísticos, con el fin de generar conocimientos que sean aprovechados por los discentes durante su formación profesional, con el desarrollo integral requerido.

Durante la formación profesional, los estudiantes perciben, interpretan, comprenden, ajustan su existencia, a partir de la toma de conciencia plena de su realidad, en sus interacciones intersubjetivas con otros pares, que tienen características diferenciadas en algunos ámbitos socioeconómicos y culturales, razón por la cual desarrollan estilos de vida variados, al mismo tiempo, enfrentan desafíos universitarios a los cuales deben responder con el compromiso y la proactividad requeridos.

Visto de este modo, el Dasein del estudiante universitario está inmerso en un ámbito formativo, con infinitas posibilidades de conocer, aprender, accionar y desarrollarse hasta alcanzar su formación personal, social y profesional para posteriormente ingresar al campo laboral.

Desde esta perspectiva los estudiantes universitarios, además de apropiarse de la realidad académica para transformarse y convertirse en profesionales idóneos, adquieren competencias para asumir su vida con sabiduría, capaces de aprender a aprender, con sentido de responsabilidad, solidaridad social y respeto por su entorno. 
La hermeneusis de la ontología del estudiante universitario, los lleva a enfrentar retos y desafíos para lograr un adecuado desempeño estudiantil, que posteriormente los conducirá a alcanzar el logro académico deseado. Para ello, deben contar con recursos didácticos actualizados, espacios físicos idóneos y principalmente con profesores actualizados con vocación de servicio que laboren en un apropiado ambiente de enseñanza-aprendizaje donde promuevan en los discentes el autoconocimiento y la autovaloración para que se reconozcan como personas, ciudadanos, como futuros profesionales y de esta manera logren alcanzar la autorrealización plena durante su formación profesional.

La universidad debe adecuarse a las exigencias de transformación tecno- científico-humanístico que nos envuelve, sobre todo en la construcción de sendas de convivencia que garanticen el proceso formativo, el respeto a la dignidad de los estudiantes como personas que deben desplegar su realización plena, no sólo en los ámbitos académicos, sino también en las interacciones con su entorno y posteriormente en su participación sociolaboral.

Finalmente, la hermeneusis de la ontología del estudiante universitario, puede entenderse como una formación académica integral que conlleva a desarrollar la autonomía para realizar las actividades que le permitan un ejercicio profesional, con espontaneidad, independencia y firmeza de carácter, hacia el cumplimiento de sus metas personales que contribuyan a las soluciones que demanda la sociedad contemporánea.

\section{Referencias}

Agencia para la Calidad del Sistema Universitario de Cataluña (2003). Educación Superior y Trabajo de Cataluña. Barcelona: AQU.

Echeverría, R. (2013). Mirada Ontológica. [Video en línea]. Recuperado de: https://www.youtube.com/watch?v=wTilkipL7qc 
Gadamer, H. (2004). Verdad y Método II. España: Sígueme.

Heidegger, M. (2005). Ser y tiempo. [Traducción de la versión alemana de 1972 por Jorge Eduardo Rivera]. Santiago de Chile: Universitaria.

Husserl, E. (1997). Ideas Relativas a una Fenomenología Pura y una Filosofía Fenomenológica. [Traducción de José Gaos]. México: Fondo de Cultura Económica.

Ji, L. (2011). Por un Mundo Armonioso. UNESCO. Recuperado de: http://unesdoc.unesco.org/images/0021/002130/213061s.pdf

Juárez, F. (2011). El Concepto de Salud: Una Explicación sobre su Unicidad, Multiplicidad y los Modelos de Salud. International Journal of Psychological Research.

López P. (2013). Fundamentos Epistémicos del Liderazgo Estudiantil. [Documento en Línea]. Recuperado de:

http://www.facso.uchile.cl/publicaciones/moebio/47/lopez.html

Malavé de Zabala, I. (2001). Características Psicosociales del Estudiante Exitoso de la Carrera Ingeniería Industrial en la Universidad Nacional Abierta. Centro Local Lara. Revista Electrónica de Investigación Científica, Humanística y Tecnológica, Volumen 2, Fascículo 3.

Morín, E. (2000). Los Siete Saberes Necesarios a la Educación del Futuro. Caracas: Face/UCV.UNESCO/CIPOST.

Organización de las Naciones Unidas para la Educación la Ciencia y la Cultura (2005). Informe de Seguimiento de Educación para Todos en el Mundo. Chile: UNESCO.

Perales, A., Mendoza, A., y Ortiz, P. (2000). El Mercado Profesional como Determinante de Inconducta Médica. Unidad de Investigación, Unidad de Postgrado y Sección Doctoral de la Unidad de Post Grado. Facultad de Medicina. Universidad Nacional Mayor de San Marcos, UNMSM Lima, Perú. Recuperado de: 
http://sisbib.unmsm.edu.pe/bvrevistas/anales/v61 n3/pdf/a06v61n3.pd

f

Salas R. (2000). La calidad en el desarrollo profesional: avances y desafíos. Ministerio de Salud Pública. Cuba: Centro Nacional de Perfeccionamiento Médico.

Silas, J. (2011). Percepción de los Estudiantes de Nivel Medio Superior sobre la Educación Superior. Dos Ciudades y Cinco Instituciones, nro. 38. [Artículo en línea]. Recuperado de:

http://www.scielo.org.mx/pdf/sine/n38/n38a10.pdf

Schwandt, T. (1997). Tres Instancias Epistemológicas para la Investigación Cualitativa. Interpretativismo, Hermenéutica y Construccionismo Social. Material traducido para fines didácticos por Lic. Indira Paz

Ugas Fermín, G. (2013). Del acto de Conocer al Discurso que lo Narra. Una Problemática Epistemológica. San Cristóbal, Táchira, Venezuela: Lito-Formas. 


\section{Mariela Eduvigis Jiménez Campos \\ e-mail: mjimenez@ucla.edu.ve}

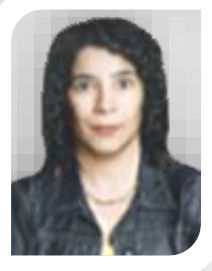

Nacida en Barquisimeto, Estado Lara, Venezuela, el 08 de octubre de 1967. Profesora de Lenguas Extranjeras, mención: Inglés, Universidad Pedagógica Experimental Libertador, UPEL-IPB (1992). Maestría en la Enseñanza del Inglés como Lengua Extranjera, UPEL-IPB (2003). Doctorante en Ciencias de la Educación en la Universidad Fermín Toro (UFT) de Cabudare, Estado Lara. Profesora de Inglés en el Colegio Inmaculada Concepción de Barquisimeto (1992-2003), Docente de Inglés en la academia Piccadilly English Center, Grupo Aeronáutico Lara y en empresas como: Cargill de Venezuela, BOC Gases, Procter \& Gamble. Docente de pregrado y postgrado en UCLA, UPEL-IPB, UPTAEB, UNEXPO. Profesora ordinaria a tiempo completo de pregrado en el Decanato de Ciencias y Tecnología (DCyT) UCLA y de postgrado en los Decanatos de Veterinaria, Agronomía y DCyT de la UCLA, desde el 2003 hasta la fecha. Actualmente, Coordinadora de Cultura del DCyT-UCLA, Coordinadora del Área de Inglés del DCyT-UCLA, Miembro del Consejo de Investigación del DCyT-UCLA. Así como, Jurado de trabajos de investigación, ponente y coautora de libros para la enseñanza del inglés, titulados: "TIME OFF 1", "TIME OFF 2" y "TIME OFF 3".

El contenido de este manuscrito se difunde bajo una Licencia de Creative Commons ReconocimientoNoComercial-Compartirlgual 4.0 Internacional 